\title{
CX. STUDIES ON HEMICELLULOSES.
}

\section{THE HEMICELLULOSES OF MAIZE COBS.}

\author{
By ISAAC ARTHUR PREECE. \\ From the Department of Biochemistry, University of Birmingham.
}

(Received June 26th, 1930.)

\section{INTRODUCTION.}

MAIZE cobs have long been recognised as a source of xylose, which may be obtained from them in a pure condition and in fairly high yield. More recently, a method for the preparation of xylose from this source has been described by Ling and Nanji [1923], who obtained the sugar in 10-12\% yield.

This xylose arises, at least in part, by the hydrolysis of hemicelluloses present in the cobs. Johnson [1896] investigated the nature of maize cob hemicellulose. After a preliminary treatment with dilute ammonia, the cobs were extracted with sodium hydroxide solution, the hemicellulose being precipitated by addition of alcohol. The product yielded xylose on hydrolysis, other sugars not being detected, while as a result of elementary analysis the formula $\left(\mathrm{C}_{5} \mathrm{H}_{8} \mathrm{O}_{4}\right)_{n}$ was ascribed to it.

Maize cob hemicellulose was thus regarded as a single substance, indeed as a pure xylan.

However, it has been indicated in a previous paper dealing with the hemicelluloses of wheat bran [Norris and Preece, 1930] that in some cases at least it may be possible to separate the hemicellulose from a given source into a number of fractions, having constitutions which are similar but, nevertheless, distinct. Moreover, it has been shown that uronic acid residues are widespread in hemicelluloses [O'Dwyer, 1926; Norman, 1929].

It was felt, therefore, that the method of separation successfully applied in the case of the hemicelluloses of wheat bran might to advantage be attempted with the hemicelluloses of maize cobs, with the added object of detecting the presence of constituents other than xylan. In a substance of such complexity as a hemicellulose, the substitution of one or more residues of xylose by residues of other sugars, or sugar derivatives, would but slightly alter its elementary composition. Thus, the analysis of maize cob hemicellulose might still approximate very closely to that for a pure xylan, even though xylose were not the only sugar obtainable from it on hydrolysis. 


\section{EXPERIMENTAL.}

\section{Preparation and purification of the hemicelluloses.}

As a preliminary to chemical treatment, the cobs were mechanically reduced to a coarse sawdust. The preparation of the hemicelluloses was carried out in exactly the same way as has been described for the preparation of the hemicelluloses of wheat bran [Norris and Preece, 1930]. The method there described again gave three fractions, designated hemicelluloses A, B and C.

Furfuraldehyde determinations were carried out on the products, and the results obtained (calculated on the dry, ash-free material) are quoted in Table I where the yields from $400 \mathrm{~g}$. of the ground cobs are also given.

Table I.

$\begin{array}{ccc}\text { Yield of crude } & \begin{array}{c}\text { Furfural- } \\ \text { product from } \\ \text { dehyde }\end{array} \\ \text { Fraction } & 400 \mathrm{~g} \text {. of cobs (g.) } & \% \\ \text { A } & 32 & 60 \cdot 70 \\ \text { B } & 24 & 57 \cdot 82 \\ \text { C } & 6 & 50 \cdot 77\end{array}$

Hemicellulose $\mathrm{A}$ was purified by dissolving in $4 \%$ sodium hydroxide solution, filtering through paper pulp, and precipitating with Fehling's solution as the copper complex. This was decomposed with dilute hydrochloric acid, and the hemicellulose precipitated and washed with acetone.

An attempt was made to separate hemicellulose $B$ into two fractions analogous to hemicelluloses B 1 and B 2 of wheat bran. However, the whole of this fraction was directly precipitated from alkaline solution by Fehling's solution; consequently this product is described as hemicellulose B 1 .

On the other hand, hemicellulose $C$ was split up by copper treatment, a portion-hemicellulose C l-being directly precipitated by Fehling's solution, while hemicellulose $\mathrm{C} 2$ was precipitated from the filtrate from this on addition of half a volume of acetone. Both fractions were further purified by a repetition of the treatment.

Thus, the hemicelluloses of maize cobs, like those of wheat bran, may be separated into four fractions, the two sets having, however, somewhat different characteristics. Thus, while under the present system of nomenclature the wheat bran-hemicelluloses are designated A, B 1, B 2 and C 2, those of maize cobs are A, B 1, C 1 and $\mathrm{C} 2$.

\section{General properties of the hemicelluloses.}

The four hemicelluloses are white, amorphous, non-reducing powders. Fraction A is insoluble in hot water; B 1 swells up in cold water, and is completely dissolved on boiling; $\mathrm{C} 1$ is partially dissolved by cold water, complete solution being obtained on boiling; $\mathrm{C} 2$ shows gum-like swelling in cold water; hot water dissolves it entirely. None of these hemicelluloses gives an iodine coloration. 
During the estimation of furfuraldehyde, it was found that the phloroglucide precipitates obtained from hemicelluloses $\mathrm{B}, \mathrm{C} 1$ and $\mathrm{C} 2$ contained appreciable amounts of a substance soluble in $95 \%$ alcohol to a red-brown solution, indicating the presence of methylpentosan in these hemicelluloses. Consequently, determinations of methylpentosan in presence of pentosan were carried out according to the method of Tollens and Ellett [see Browne, 1912]. The percentages of methylpentosan found and the percentage yields of furfuraldehyde are given in Table II. Carbon dioxide numbers were also obtained by the method of Nanji, Paton, and Ling [1925]. The rotatory powers of A, B 1 and $\mathrm{C} 1$ in $4 \%$ sodium hydroxide solution were also determined.

All the results given in this table are calculated on the dry, ash-free hemicelluloses, and in each case the figures quoted are the mean for a number of closely agreeing determinations.

Table II.

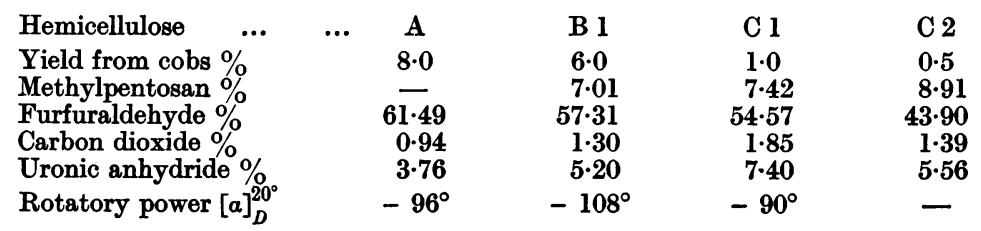

Since hemicellulose C, before separation into its constituents, was found to give a furfuraldehyde yield of $50.77 \%$, its components $\mathrm{C} 1$ and $\mathrm{C} 2$ are present in the ratio of two parts of the former to one of the latter.

\section{Hydrolysis.}

Hemicellulose $A$. Some $5 \mathrm{~g}$. of the hemicellulose were hydrolysed by heating under reflux for 4 hours with $250 \mathrm{cc}$. of $1 \%$ sulphuric acid. The sulphuric acid was then neutralised by the addition of the requisite volume of saturated barium hydroxide solution, and the precipitated barium sulphate filtered off. The filtrate was evaporated in vacuo just to dryness, and the residue extracted twice with $50 \mathrm{cc}$. portions of absolute alcohol. The alcoholic extracts after filtering were united, the alcohol removed by evaporation, and drying completed in vacuo. The residue insoluble in absolute alcohol was twice extracted with 50 cc. portions of boiling $80 \%$ alcohol, the united extracts being taken to dryness as before. The amount of residue after $80 \%$ alcohol extraction was extremely small, and was neglected.

The bulk of the hydrolysis product was found to have passed into the absolute alcohol. The sugar of this fraction gave $[\alpha]_{D}^{20^{\circ}}=+18^{\circ}$, and melted after recrystallisation at $139^{\circ}$.

From a portion of this sugar an osazone was prepared. The product was pure xylosazone, having the characteristic crystalline appearance and melting after recrystallisation at $160^{\circ}$. The presence of xylose was confirmed by the preparation of the characteristic cadmium xylonobromide. 
From the sugar soluble in $80 \%$ alcohol a mixture of osazones was obtained. One, insoluble in hot water, had a crystalline appearance similar to that observed in the case of insoluble phenylhydrazine derivatives obtained from the hydrolysis liquors of certain of the hemicelluloses of wheat bran [Norris and Preece, 1930]. After purification it melted sharply at $160^{\circ}$. In all probability, this product is a derivative of glycuronic acid. The second osazone was that of xylose, and the presence of this sugar was confirmed by the preparation of cadmium xylonobromide.

Sugars other than xylose could not be detected in either of the two fractions.

In a second hydrolysis, a weight equivalent to $1.549 \mathrm{~g}$. of dry, ash-free hemicellulose $\mathrm{A}$ was treated with $1 \%$ sulphuric acid in the usual way, the sulphuric acid neutralised with the requisite volume of barium hydroxide solution, and the barium sulphate removed by filtration. The precipitate was carefully washed with water, and the filtrate and washings combined. The liquid still remained acid to phenolphthalein, and a further amount of barium hydroxide was added to bring the reaction to the neutral point. After evaporation to dryness in vacuo, the sugar was extracted several times with small quantities of boiling absolute alcohol, and the extracts filtered hot, and combined.

From the combined extracts there was recovered $1.45 \mathrm{~g}$. of xylose, giving for $c=3 \cdot 362[\alpha]_{D}^{20^{\circ}}=+19 \cdot 5^{\circ}$. It is shown below that hemicellulose A contains $94.36 \%$ of xylan, so that the present amount of xylose obtained represents some $88 \%$ of the theoretical yield.

Hemicellulose B 1. The neutral hydrolysis liquor from hemicellulose B 1 was taken to dryness in vacuo and extracted successively with absolute alcohol and with $80 \%$ alcohol. The bulk of the sugar was again found to pass into the absolute alcohol.

The sugar soluble in absolute alcohol gave $[\alpha]_{D}^{20^{\circ}}=+18^{\circ}$. An osazone prepared from it proved to be xylosazone, melting after recrystallisation at $158^{\circ}$. Cadmium xylonobromide was also prepared.

The product soluble in $80 \%$ alcohol showed $[\alpha]_{D}^{20^{\circ}}=+19^{\circ}$. This also was xylose, yielding xylosazone melting at $158^{\circ}$, and yielding cadmium xylonobromide.

Hydrolysis products other than xylose could not be detected in either fraction, nor was a phenylhydrazine derivative insoluble in hot water obtained from either.

A hydrolysis on quantitative lines was carried out in a manner similar to that employed in the case of the second hydrolysis of hemicellulose A described above.

$1.72 \mathrm{~g}$. of hemicellulose B 1 yielded $1.58 \mathrm{~g}$. of xylose, showing for $c=2 \cdot 31[\alpha]_{D}^{20^{\circ}}=+19^{\circ}$, and melting at $140-141^{\circ}$. This represents a yield of xylose approximating to $92 \%$ of the theoretical, since, as is shown below, hemicellulose B 1 contains $87.60 \%$ of xylan. 
Hemicelluloses $C 1$ and $C 2$. Owing to the small amounts of these products available for examination, only preliminary investigations of the nature of the sugars arising on hydrolysis could be attempted. However, these sufficed to indicate with certainty the nature of the pentoses produced.

To the hydrolysis liquor obtained from $1 \mathrm{~g}$. of hemicellulose $\mathrm{C} 1$ saturated barium hydroxide solution was added until the liquid remained just acid to phenolphthalein. The filtrate from barium sulphate was concentrated to a bulk of $50 \mathrm{cc}$. and divided into several portions for examination.

An osazone was prepared. The product was mainly pentosazone, soluble in hot water. This had the crystalline form of xylosazone and melted after several recrystallisations at $154^{\circ}$, a somewhat low temperature. The small amount of derivative insoluble in hot water had the crystalline form associated with the phenylhydrazine derivative of glycuronic acid. It was not further examined.

The presence of xylose was confirmed by the preparation from a second portion of hydrolysis liquor of the characteristic cadmium xylonobromide. Arabinose and hexoses were absent.

The hydrolysis liquor from $1 \mathrm{~g}$. of $\mathrm{C} 2$ was treated similarly to the above. The osazone obtained was again a mixture. The small amount insoluble in hot water was the glycuronic acid derivative, melting after recrystallisation from dilute alcohol at $155-156^{\circ}$. The hot water-soluble osazone resembled in crystalline appearance the osazone of arabinose; it melted at $158^{\circ}$.

By treating a portion of the hydrolysis liquor in the usual way with diphenylhydrazine, a copious precipitate of colourless crystalline arabinose diphenylhydrazone was obtained on standing. After recrystallisation from $50 \%$ alcohol this gave a melting point of $200^{\circ}$. The presence of arabinose amongst the products of hydrolysis of hemicellulose C 2 was thus confirmed. Xylose and hexoses were absent.

In the case of none of the hemicelluloses was it possible to identify the methylpentosan constituent. Further, the very small amounts of uronic acid residues present in all cases made their absolute characterisation impossible, though, as has been shown, glycuronic acid appears to be present in at least three cases.

\section{Discussion.}

It has been shown by Cunningham and Dorée [1914] and other workers, that $\omega$-hydroxymethylfurfuraldehyde gives with phloroglucinol a precipitate which is soluble in alcohol. However, it has been shown that the hemicelluloses of the present research contain no hexosan, and it thus seems legitimate to regard the alcohol-soluble portion of the phloroglucide precipitate as due solely to methylpentosans.

From the results of the hydrolyses, and from the analytical data quoted in Table II, it is possible to calculate with fair accuracy the relative proportions of xylan, araban, methylpentosan, and uronic anhydride present in the four fractions. Use is made of the following relations: 
100 g. uronic anhydride yield $16 \cdot 7$ g. furfuraldehyde.

$100 \mathrm{~g}$. xylan yield $64 \cdot 5 \mathrm{~g}$. furfuraldehyde.

$100 \mathrm{~g}$. araban yield $53.5 \mathrm{~g}$. furfuraldehyde.

The calculated values are given in Table III.

\section{Table III.}

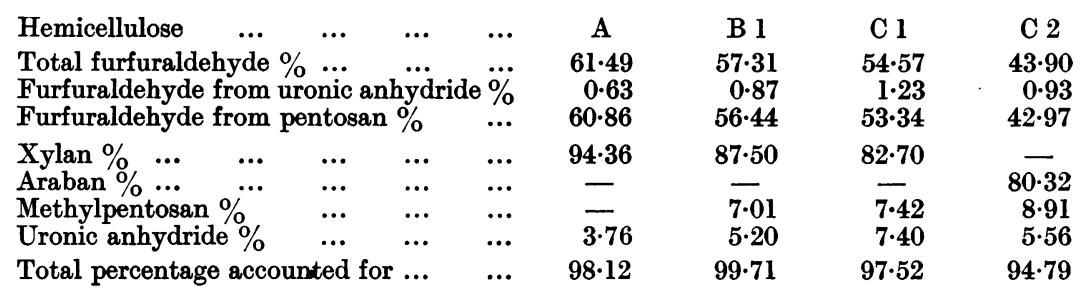

Thus, it has been possible in all cases to account satisfactorily for practically $100 \%$ of the constituents. It will be seen that hemicelluloses A, B 1 and $\mathrm{C} 1$ form a series, in which increasing solubility in water is accompanied by simultaneous increases in the amounts of uronic anhydride and methylpentosan present, with corresponding decreases in the xylan percentages. However, the corresponding rotatory powers (Table II) do not show a similar progressive change.

The simultaneous occurrence in some cases of methylpentosan and uronic anhydride is of interest; this might have been expected, however, since these may be regarded as derived from hexosan by reduction and oxidation respectively.

If the above fractions represent individual hemicelluloses, it is apparent that the hemicellulose molecule is one of considerable complexity. Thus, fraction B 1, yielding $57.31 \%$ furfuraldehyde, $7.01 \%$ methylpentosan, and $5 \cdot 20 \%$ uronic anhydride, would correspond to a molecule of the minimum dimensions

$$
\left(\mathrm{C}_{5} \mathrm{H}_{8} \mathrm{O}_{4}\right)_{43} \cdot\left(\mathrm{C}_{6} \mathrm{H}_{10} \mathrm{O}_{4}\right)_{3} \cdot\left(\mathrm{C}_{6} \mathrm{H}_{8} \mathrm{O}_{6}\right)_{2}
$$

with a molecular weight of approximately 6500 . Such a structure would give a furfuraldehyde yield of $57.47 \%$ and would contain $6.8 \%$ and $5.4 \%$ of methylpentosan and uronic anhydride respectively.

The alternative view that the fractions isolated represent aggregates of molecules of similar physical properties has much to commend it, and at present it is manifestly unwise to claim that individual hemicelluloses have been isolated.

\section{SUMmary.}

1. The hemicellulose of maize cobs has been separated into four fractions, none of which corresponds to a pure xylan.

2. Hemicellulose A yields on hydrolysis xylose and a uronic acid; B 1 and C 1 yield varying proportions of xylose, uronic acid, and methylpentose; C 2 gives arabinose, uronic acid, and methylpentose. 
3. In all cases the proportion of pentose residues present far exceeds the combined proportions of residues of methylpentose and uronic acid.

The author's best thanks are due to Prof. A. R. Ling and Dr F. W. Norris for their encouragement and advice.

\section{REFERENCES.}

Browne (1912). Handbook of sugar analysis (New York). Cunningham and Dorée (1914). Biochem. J. 8, 438.

Johnson (1896). J. Amer. Chem. Soc. 18, 214.

Ling and Nanji (1923). J. Chem. Soc. 123, 620.

Nanji, Paton and Ling (1925). J. Soc. Chem. Ind. 44, 253 T.

Norman (1929). Biochem. J. 23, 1353.

Norris and Preece (1930). Biochem. J. 24, 59.

O'Dwyer (1926). Biochem. J. 20, 656. 